Journal of Mathematics and Statistics 6 (2): 131-135, 2010

ISSN 1549-3644

(C) 2010 Science Publications

\title{
A New Method to Describe Image Theory for an Imperfect Conductor
}

\author{
${ }^{1}$ Scott L. Meredith, ${ }^{1}$ Susan K. Earles and ${ }^{2}$ Niescja E. Turner \\ ${ }^{1}$ Department of Electrical and Computer Engineering, \\ ${ }^{2}$ Department of Physics and Space Sciences, \\ Florida Institute of Technology, Melbourne, Florida, USA
}

\begin{abstract}
Problem statement: Modify the equations associated with image theory in order to account for perfect and imperfect conductors. Approach: A novel approach for describing the application of image theory for an imperfect conductive surface was presented. The method presented here purposely downplays the physics of how image theory was employed to account for a charge which is in the presence of an imperfect conductive surface. In turn, it adopted an approach which focused on the geometry that existed between the charged particle and surface ground. In doing so, the proposed method formulated a solution that had minimized the complexity of the original problem while providing an approximation founded upon a geometric relationship. Results: The equations derived had elicited the concept of using plane geometry to augment image theory. Conclusion: A method for evaluating image theory for the imperfect conductor had been presented. As the results had shown, the equations derived had provided an augmented approach to account for surfaces which were both perfect and imperfect.
\end{abstract}

Key words: Image theory, degraded image, electromagnetic fields and imperfect conductors

\section{INTRODUCTION}

Image theory, in its current form, assumes that an imaged charge is in the presence of perfect conductor. By assuming the material is a "perfect conductor" allows one to account for all of the charge constituents. Therefore, one can assume that a charge over an infinitely conductive ground has a perfect mirror image. This "mirror image" can be quantified by taking the charge's spatial coordinates which are perpendicular to the surface and rotating or projecting them by $180^{\circ}$ (Balanis, 1989). Taking the cosine of this angle gives rise to an image charge that is equal in magnitude but opposite in polarity. However, in reality the surface in the presence of a charged particle is not a perfect conductor. With this in mind, one must presume that formulas which leverage this "perfect conductor" assumption will loss accuracy as the surface becomes increasing non-conductive. For the purposes of this study, we introduce and modify an approach originally proposed by Meredith and Earles (2010) to develop equations which account for both perfect and imperfect surfaces. In doing so, this approach has downplayed the physics of image theory in order to develop a solution which has minimized the complexity of the original problem.

\section{MATERIALS AND METHODS}

The electric field $\mathrm{E}$ is defined as the force per unit charge. Equation 1 describes the electric field as it would be experienced by the small stationary charge $\mathrm{q}_{0}$ (Jackson, 1999):

$$
E=\frac{F}{q_{0}}
$$

Where:

$\mathrm{F}=$ The force experience by the stationary test charge

$\mathrm{E}=$ The field wherein the particle is located

A conductor by definition, contain charges capable of moving freely under the action of an applied electric field. In principle, this states that the strength of the electric field dictates the speed at which the charge particles will travel within the conductive material. Furthermore if charged particles can no longer be accelerated, then the electric field must equal zero. Given a basic expression between an electric field and particle has been described, one can now expand this idea to consider the effects of multiple charges.

Coulombs law states that the magnitude of the electrostatic force between two point charges is directly

Corresponding Author: Scott L. Meredith, Department of Electrical and Computer Engineering, Florida Institute of Technology, Melbourne, Florida, USA 
proportional to the product of the magnitudes of each of the charges and inversely proportional to the square of the total distance between them. We can now expand (1) to include more than one charge such that:

$$
\mathrm{F}=\mathrm{k}_{\mathrm{e}} \frac{\mathrm{q}_{0} \mathrm{q}}{\mathrm{x}^{2}}
$$

where, $\mathrm{K}_{\mathrm{e}}$, known as Coulombs constant, is a proportionality constant whose value is determined by the medium that the charged objects are immersed in and $\mathrm{x}$ is the distance between the charges $\mathrm{q}_{0}$ and $\mathrm{q}$. When the medium is air, this value is approximately $8.987 \times 10^{9} \mathrm{Nm}^{2} \mathrm{C}^{-2}$, which is calculated using $\left(4 \pi \varepsilon_{0}\right)^{-1}$ where $\varepsilon_{0}=1 / \mu_{0} c$, with the permeability of free space $\mu_{0}$, the permittivity of free space $\varepsilon_{0}$ and the speed of light c.

One can expand Eq. 1 to derive an expression that equates the potential as a function of charge. By rearranging (1) in terms of $\mathrm{F}$ and equating it to (2) one would obtain:

$$
\mathrm{E}=\frac{\mathrm{k}_{\mathrm{e}} \mathrm{q}}{\mathrm{x}^{2}}
$$

With the electric field being written in terms of the potential as:

$$
\mathrm{E}=\frac{\Phi}{\mathrm{x}}
$$

One can use Eq. 3 and 4 to solve for the potential in terms of charge such that:

$$
\Phi(\mathrm{x})=\frac{\mathrm{k}_{\mathrm{e}} \mathrm{q}}{\mathrm{x}}
$$

However, let's consider the case of a charged particle which is located above a conductive surface. In order to describe the magnitude of the electric field at some arbitrary point $\mathrm{P}$, one must develop an equation which describes the relationship between the charged particle above a conductive surface.

From Fig. 1 we can expand (5) to account for the distance between the charge $\mathrm{q}$ and vector $\mathrm{x}$ such that:

$$
\Phi(\mathrm{x})=\frac{\mathrm{k}_{\mathrm{e}} \mathrm{q}}{|\overline{\mathrm{x}}-\overline{\mathrm{y}}|}
$$

As previously noted, a conductor contains charges capable of freely moving in the presence of an electric field. In the literature, image theory has been employed to describe the relationship between a charged particle and a perfectly conductive ground. We can illustrate the method of images as shown in Fig. 1 by considering the problem for a point charge $q$ located at $y$ relative to the origin, around which is centered a grounded conducting sphere of radius a (Jackson, 1999).

One could think about image theory this way. Let's suppose you have a coherent light source and you shine it upon a dingy piece of metal. Some of the light will reflect back towards you but much of it will be lost due to refraction and/or absorption. Now take the same coherent light source and shine it towards a highly reflective mirror. You'll notice that most of the light, about $99 \%$, will reflect back while only $\sim 1 \%$ is lost due to refraction and/or absorption. Using the highly reflective mirror allowed you to account for the majority of light. That is, you're cognizant of where the light went because it reflected back towards you. In essence, by assuming we have a perfectly conducting material that acts like a mirror allows one to project a particles image $180^{\circ}$ (Balanis, 1989) from the radial position where the original particle lies with respect to the conductive surface. This idea is illustrated in Fig. 2 (Jackson, 1999).

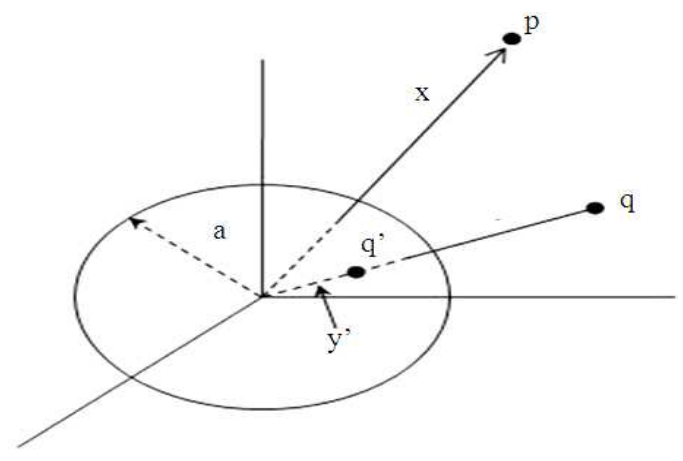

Fig. 1: Conducting sphere of radius a, with charge $q$ and image charge, q'

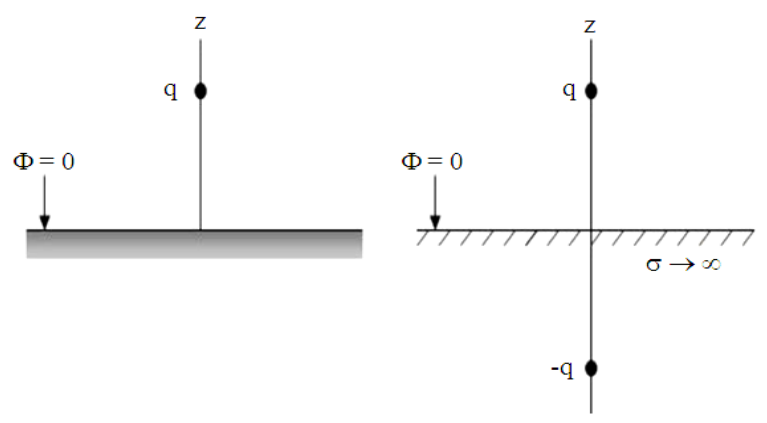

Fig. 2: Solution by method of images 


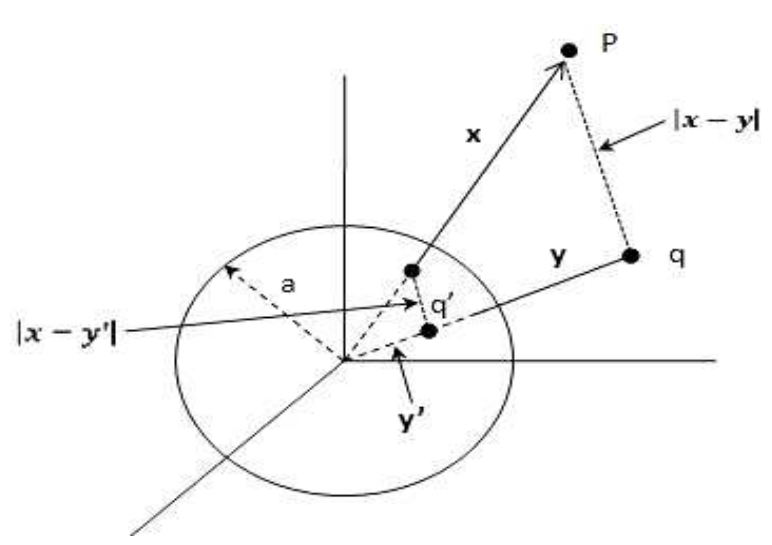

Fig. 3: The magnitude of the distances associated with the incident charge and image charge

Figure 2 assumes that we're taking a very small slice of the spherical surface which houses the imaged charge as shown in Fig. 1. If this slice is small enough it can be modeled by a flat surface.

Given Fig. 3, we can expand (6) to account for the image charge q' such that:

$\Phi(\mathrm{x})=\frac{\mathrm{k}_{\mathrm{e}} \mathrm{q}}{|\overline{\mathrm{x}}-\overline{\mathrm{y}}|}+\frac{\mathrm{k}_{\mathrm{e}} \mathrm{q}^{\prime}}{|\overline{\mathrm{x}}-\overline{\mathrm{y}}|}$

where, $|\bar{x}-\bar{y}|$ and $|\bar{x}-\bar{y}|$ are the distances that exists between the charge $\mathrm{q}$ and image charge q' with respect to the unit vector $\mathrm{x}$.

In order to augment the approach used from image theory, this study will introduce the idea of the degraded image. This ideology accommodates both perfect and imperfect projected images. With traditional image theory, it assumes that a charge in the presence of a perfect conductor has a mirror image. However, as the surface below this charged particle becomes less conductive, one can no longer assume that its image remains unchanged. In fact, one must concede to the idea that the entire image can no longer be projected in the same fashion as the image of a charge in the presence of a perfect conductor. With the adoption of this idea in place, it's logical to presume that as the surface below the charged particle becomes less conductive, the image charge will become degraded as shown in Fig. 4. Given the image charge has become degraded, some of the charge constituents have now become displaced. Since the conservation of charge must be preserved, these dislocated constituents will now collect along the surface of the conductive material.

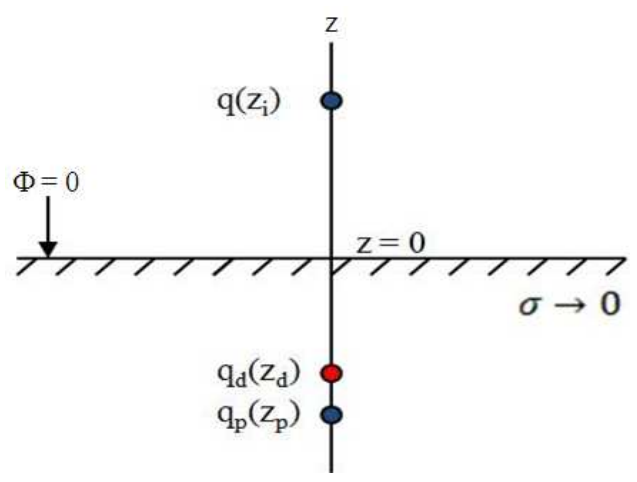

Fig. 4: Application of the degraded image

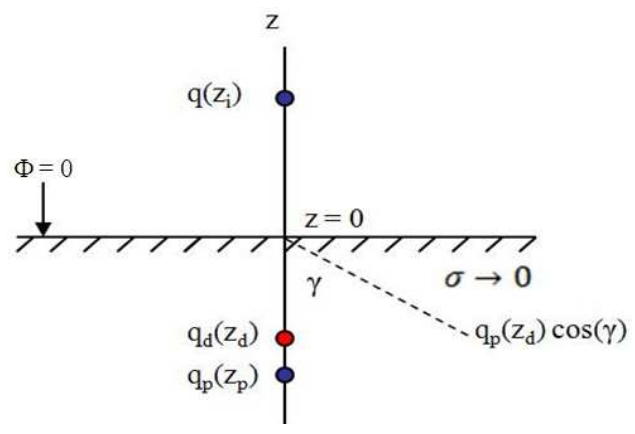

Fig. 5: The projection of a perfect image's magnitude

Figure 4 illustrates the how the degraded image charge, $\mathrm{q}_{\mathrm{d}}$ will change with respect to the perfect image charge, $\mathrm{q}_{\mathrm{p}}$ as the conductivity, $\sigma$ of the surface decreases. As the surface becomes a perfect insulator, the magnitude of the degraded image charge approaches zero. This methodology has purposely downplayed the physics of how image theory is employed to account for a charge which is in the presence of an imperfect conductive surface. In doing so, this model formulates a solution that has minimized the complexity of the original problem while providing an approximation founded upon a geometric relationship. Knowing how the image charge is affected by the surface conductivity allows one to develop an equivalency between the two by exploiting the geometry of Fig. 4. Since the contribution from the magnitude of the perfect image charge is generally known, this value can be scaled to account for a changing conductivity as shown in Fig. 5.

As Fig. 5 illustrates, the contribution from the degraded charge can be quantified by taking the magnitude of the perfect image charge and scaling it by a factor which accounts for the loss. This can be realized by taking the projection of the perfect image's magnitude and rotating it along the $\mathrm{z}=0$ axis until it 
shares the same $\mathrm{z}$ component as the degraded image. Do so will allow one to associate the perfect image with the degraded image by multiplying the scaling factor, $\cos (\gamma)$. Therefore, we can write the degraded charge in terms of the perfect charge such that:

$\mathrm{q}_{\mathrm{d}}=\mathrm{q}^{\prime} \cos (\gamma)$

where, $q_{d}$ is the degraded image charge and the cosine of the angle, $\gamma$ accounts for the loss. In order to maintain the conservation of charge, one must arrive at the notion that the image charge constituents that are no longer present within the degraded image must now be present elsewhere. With this in mind, we can infer that these image charge constituents will now collect along the surface. Although these surface charge constituents spread out radially, their contributions can be approximated by formulating two distinct charges, each of which lie on opposite sides of the z-to-ground plane interface. In principle, these two surface charges represent the summation of each of their respective radial constituents, thus formulating a viable approximation to those present. We can describe the total surface charge with the following:

$\mathrm{q}_{\mathrm{s}}=\mathrm{q}^{\prime}(1-\cos (\gamma))$

\section{RESULTS}

In order to maintain symmetry, we can write a general expression for the potential at $\mathrm{z}=0$ such that:

$\Phi(\mathrm{x}, \mathrm{z}=0)=0=\Phi\left(\mathrm{x}, \mathrm{z}=\mathrm{z}_{\mathrm{i}}\right)+\Phi\left(\mathrm{x}, \mathrm{z}=\mathrm{z}_{\mathrm{p}}\right)$

where, $\Phi\left(\mathrm{x}, \mathrm{z}=\mathrm{z}_{\mathrm{i}}\right)$ and $\Phi\left(\mathrm{x}, \mathrm{z}=\mathrm{z}_{\mathrm{p}}\right)$ are the potentials located at the incident charge and perfect image charge respectively. An alternative form of this expression can be written to include the equations associated with these charges at their respective $\mathrm{z}$ coordinates. Thus, one can now update (10) to include these equivalent terms to yield:

$\Phi(\mathrm{x}, \mathrm{z}=0)=\frac{\mathrm{q}}{|\overline{\mathrm{x}}-\overline{\mathrm{y}}|}+\frac{\mathrm{q}^{\prime}}{|\overline{\mathrm{x}}-\overline{\mathrm{y}}|}$

However, as the potential located at the image charge becomes degraded, Eq. 10 is no longer valid. In order to validate this expression, one would have to add a form of potential to the right hand side of the equation. This term is made up of the dislocated charge constituents who have now collected along the surface of the conductive material. The inclusion of this surface charge now brings the needed symmetry to the equation and thus preserves the conservation of charge. The potential of the perfect image charge can be written as:

$\Phi\left(\mathrm{x}, \mathrm{z}=\mathrm{z}_{\mathrm{p}}\right)=\Phi\left(\mathrm{x}, \mathrm{z}=\mathrm{z}_{\mathrm{d}}\right)+\Phi\left(\mathrm{x}, \mathrm{z}=\mathrm{z}_{\mathrm{s}}\right)$

where, $\Phi\left(\mathrm{x}, \mathrm{z}=\mathrm{z}_{\mathrm{d}}\right)$ and $\Phi\left(\mathrm{x}, \mathrm{z}=\mathrm{z}_{\mathrm{s}}\right)$ are the potentials located at the degraded charge and surface charge respectively. Therefore, one can now re-write (10) to include the additional terms from (10) with:

$$
\begin{aligned}
\Phi(\mathrm{x}, \mathrm{z}=0)=0= & \Phi\left(\mathrm{x}, \mathrm{z}=\mathrm{z}_{\mathrm{i}}\right)+\Phi\left(\mathrm{x}, \mathrm{z}=\mathrm{z}_{\mathrm{d}}\right) \\
& +\Phi\left(\mathrm{x}, \mathrm{z}=\mathrm{z}_{\mathrm{s}}\right)
\end{aligned}
$$

As with (11), an alternative form of this expression can be derived by simply substituting the equations associated by the potential terms at their given $\mathrm{z}$ coordinates. In doing so, we can now write a general expression which describes both perfect and imperfect conductive surfaces with the following:

$\Phi(\mathrm{x}, \mathrm{z}=0)=\frac{\mathrm{q}}{|\overline{\mathrm{x}}-\overline{\mathrm{y}}|}+\frac{\mathrm{q}_{\mathrm{d}}}{|\overline{\mathrm{x}}-\overline{\mathrm{y}}|}+\frac{\mathrm{q}_{\mathrm{s}}}{|\overline{\mathrm{x}}-\overline{\mathrm{y}}|}$

In principle, if it is assumed the conductivity of the surface approaches infinity, the charge-to-ground distance y and perfect image charge-to-ground distance $y$ ' are equivalent. However as the surface becomes less conductive, a portion of the image charge now becomes distributed along the surface as indicated in (13). Since a charge located at the surface no longer relies upon the vector y', one should presume its contribution to be negligible. With this in mind, one can now re-write (14) to include this change along with (8) and (9) to describe the final expression written as:

$\Phi(\mathrm{x}, \mathrm{z}=0)=\frac{\mathrm{q}}{|\overline{\mathrm{x}}-\overline{\mathrm{y}}|}+\frac{\mathrm{q}^{\prime} \cos (\gamma)}{\left|\overline{\mathrm{x}}-\overline{\mathrm{y}}^{\prime}\right|}+\frac{\mathrm{q}^{\prime}(1-\cos (\gamma))}{|\overline{\mathrm{x}}|}$

\section{DISCUSSION}

In Eq. 15, we utilized a novel approach to augment image theory. Surmising the image charge vertical position can vary as a function of surface conductivity aided in the development of this formula. This methodology has greatly simplified how image theory could be employed for surfaces which are no longer consider perfect conductors. In doing so, an alternative 
method to image theory has been presented which leverages the variability of the image charge with surface conductivity.

\section{CONCLUSION}

A method for evaluating image theory for the imperfect conductor has been presented. As the results have shown, the equations derived have provided an augmented approach to account for surfaces which are both perfect and imperfect. The methodology presented here has purposely downplayed the physics of how image theory is employed to account for a charge which is in the presence of an imperfect conductor. In turn, it adopted an approach which focused on the geometry that exists between the charged particle and surface ground. In doing so, the proposed method formulated a solution that has minimized the complexity of the original problem while providing an approximation founded upon a geometric relationship.

\section{REFERENCES}

Balanis, C.A., 1989. Advanced Engineering Electromagnetics. John Wiley and Sons Inc., New Jersey, USA, ISBN: 0-471-62194-3, pp: 1008.

Jackson, J.D., 1999. Classical Electrodynamics. 3rd Edn., John Wiley and Sons Inc, New Jersey, USA., ISBN: 0-471-30932-X, pp: 808.

Meredith, S.L and S.K. Earles, 2010. Electromagnetic fields induced by lightning striking a perfect and imperfect conductive surface. Proceeding of the 21st International Lightning Detection Conference, (to appear), Apr. 2010, Orlando, FL. USA. 